\section{Drivers of metastasis}

Neutrophils are important in inflammation, but their contribution to tumorigenesis has remained unclear. In Nature, Wculek and Malanchi show that neutrophils are the main drivers of metastatic invasion of the lungs in a mouse model of breast cancer. CD11 $b^{+}$Ly $6 G+^{+}$neutrophils are present at low frequencies in the primary tumors, but they accumulate in the pre-metastatic lung before infiltration by cancer cells, and their numbers increase in the metastatic lung. Genetic, conditional or antibody-mediated depletion of neutrophils at the pre-metastatic stage diminishes metastatic progression with no effect on the primary tumors. Premetastatic lung neutrophils isolated ex vivo and neutrophil-derived leukotriene mediators enhance the metastatic initiation potential of cancer cells, possibly by providing a proliferative advantage to highly tumorigenic cells. Genetic or pharmacological deletion of leukotrienes diminishes spontaneous lung metastasis in mice. IV Nature (9 December 2015) doi:10.1038/nature 16140

\section{CCR7 polysialylation}

Leukocytes use the chemokine receptor CCR7 to traffic from interstitial tissues into lymphatics and draining lymph nodes, in response to the chemokines CCL19 and CCL21. In Science, Kiermaier et al. reveal that polysialylation of CCR7 regulates dendritic cell (DC) trafficking. Mice lacking the polysialyltransferase ST8Sia IV fail to post-translationally modify CCR7 and have diminished lymph node cellularity. ST8Sia IV-deficient DCs fail to migrate in response to CCL21; however, their migration to CCL19 remains unaltered. Accordingly, CCL21 exists in an auto-inhibited conformation. This autoinhibition is relieved by interaction with polysialyl carbohydrate chains of CCR7, which allows ST8Sia IV-sufficient DCs to respond to CCL21. Further investigation is warranted to understand why such regulatory mechanisms arose to 'fine-tune' DC migration and how this modification is altered during infection or other disease states.

$L A D$

Science (10 December 2015) doi:10.1126/science.aad0512

\section{Coinfection enhances inflammation}

Helminth infections tend to be endemic in regions with a high incidence of pulmonary tuberculosis and have a modulatory effect on immune responses to Mycobacteria tuberculosis. In the Journal of Clinical Investigation, Khader and colleagues assess mice coinfected with the helminth Schistosoma mansoni and M. tuberculosis. Preinfection with S. mansoni or vaccination with S. mansoni egg antigen reversibly impairs $M$. tuberculosisspecific type 1 helper T cell responses, and lung inflammation. Furthermore, latent $\boldsymbol{M}$. tuberculosis is reactivated by vaccination with $S$. mansoni egg antigen. The exacerbated lung inflammation is strongly dependent on the presence of arginase-1-positive macrophages. Finally, using outbred mice with a spectrum of $\boldsymbol{M}$. tuberculosis-dependent pulmonary inflammation, the authors show that the expression of arginase- 1 is proportional to disease severity even in the absence of helminth coinfection. These results thus shed light on how helminths can enhance the severity of tuberculosis.

\section{Sensing cholesterol dynamics}

Perturbations in lipid metabolism are observed in viral and microbial infections, while type 1 interferon signaling is known to downregulate cellular cholesterol synthesis and increase uptake of lipids from extracellular sources. In Cell, Bensinger and colleagues confirm that viral infection and type 1 interferon signaling induce a metabolic shift from de novo synthesis to import and also show that limiting the cholesterolbiosynthetic pathway induces spontaneous type 1 interferon responses and enhances antiviral immunity. Deletion of the endoplasmic reticulum chaperone SCAP or the transcription factor SREBP2, which decrease cholesterol synthesis, induces viral resistance and a type 1 interferon response in bone marrow-derived macrophages and mouse embryonic fibroblasts. The type 1 interferon response in these cells is dependent on the cGAS-STING-TBK1-IRF3 pathway and is 'rescued' by the addition of exogenous cholesterol, which suggests that the amount of cholesterol in a cell can modulate the sensitivity of STING to its ligands. Cell (10 December 2015) doi:10.1016/j.cell.2015.11.045

\section{RANK recruiting}

The blood-brain barrier restricts the entry of leukocytes into central nervous system tissues. This border consists of an endothelial layer and a glial cell layer, separated by cerebrospinal fluid. In Immunity, Guerrini et al. report that the entry of T cells into the central nervous system parenchyma requires interaction between the cytokine receptor RANK, expressed on astrocytes, and its ligand RANKL, expressed on activated T cells. RANK signaling induces astrocyte expression of the inflammatory chemokine CCL20. Mice lacking expression of RANKL on T cells or expression of RANK by astrocytes fail to develop experimental autoimmune encephalomyelitis, due to diminished production of CCL20 and vastly lower numbers of T cells that accumulate at the glial border. Similarly, pharmacological inhibition of RANKL blocks astrocyte expression of CCL20 and diminished cellular infiltration. Thus, signaling via the RANK-RANKL axis contributes to cellular migration through the blood-brain barrier and presents a potential therapeutic target for blocking such breaches. $L A D$ Immunity (15 December 2015) doi:10.1016/

j.immuni.2015.10.017

\section{Evading hyaluronan danger}

Hyaluronan (HA) is a major extracellular matrix component that, when broken down, acts as a damage-associated molecular pattern sensed by Toll-like receptor 2 (TLR2) and TLR4. In Cell Host and Microbe, Liu and colleagues demonstrate that hyaluronidases produced by certain Grampositive bacteria serve an immunoevasive function. Group B Streptococcus (GBS) hyaluronidase digests HA into disaccharides that are much smaller than those produced by host hyaluronidases. Unlike host-generated HA fragments, these HA disaccharides are not stimulatory and block signaling via TLR2 and TLR4. GBS with mutant hyaluronidase trigger greater inflammation and show diminished infectivity in vivo. In contrast to the HA fragments produced by Gram-positive pathogens, those produced by the free-living bacteria Streptomyces hyalurolyticus are still stimulatory, which probably reflects differing evolutionary pressures. Hyaluronidases derived from certain Gram-positive pathogens therefore aid evasion of the immune system and bacterial spreading in vivo.

Cell Host Microbe 18, 694-704 (2015) 